\title{
Effectiveness of a condensed protocol for disclosing APOE genotype and providing risk education for Alzheimer disease
}

\author{
J. Scott Roberts, PhD ${ }^{1}$, Clara A. Chen, MHS², Wendy R. Uhlmann, MS, CGC ${ }^{3,4}$, \\ Robert C. Green, MD, MPH ${ }^{5}$; for the REVEAL Study Group
}

Purpose: Brief, effective models of patient genetic education are needed for common, complex diseases. Using Alzheimer disease as a model, we compared participants' risk knowledge and recall in extended versus condensed education protocols.

Methods: A four-site randomized clinical trial enrolled 280 firstdegree relatives of individuals with Alzheimer disease (mean age $=58$ years, $71 \%$ female); each received lifetime Alzheimer disease risk information (range: 13-74\%) that incorporated apolipoprotein E genotype. In the condensed protocol, participants received an educational brochure in place of an in-person education session. Outcomes were assessed at 6 weeks and 6 months following risk disclosure.

Results: The condensed protocol required less clinician time than the extended protocol (mean $=34 \mathrm{~min}$ vs. $77 \mathrm{~min}$ ). The groups did not differ on recall of apolipoprotein E genotype or lifetime risk, and most participants in both groups recalled and retained this information over time. Both groups showed improvement from baseline in Alzheimer disease risk knowledge (e.g., understanding the magnitude of apolipoprotein E genotype effect on risk).

Conclusion: A condensed protocol for communicating genetic risk for Alzheimer disease achieved similar educational results as an extended protocol in this study. Further research should explore the efficacy of brief genetic education protocols for complex diseases in diverse populations.

Genet Med 2012:14(8):742-748

Key Words: Alzheimer disease; APOE; genetic counseling; health education; risk communication

increased collaboration with other health-care professionals, use of educational media, and briefer protocols., ${ }^{2,3}$

Although there is a need for more streamlined models of genetic education, to date there have not been randomized clinical trials to examine whether brief protocols can provide comparably effective education about genetic risk for common, adult-onset diseases as more time-intensive approaches. Alzheimer disease $(\mathrm{AD})$, in which a common variant of the apolipoprotein $\mathrm{E}(A P O E)$ gene is a prominent risk factor, provides a context within which to examine genetic education for complex diseases. A series of multicenter clinical trials, collectively known as the Risk Evaluation and Education for Alzheimer's Disease (REVEAL) Study, has examined the impact of providing $A P O E$-based $\mathrm{AD}$ risk assessments to first-degree relatives of patients with AD. Findings from the initial trial indicated that $A P O E$ genotype and $\mathrm{AD}$ risk information can be provided via a comprehensive education and counseling protocol without undue distress or misunderstandings. ${ }^{4-7}$ We report here on the second trial, in which we examined the efficacy of providing the same information in a more clinically feasible protocol. 


\section{Study overview}

\section{MATERIALS AND METHODS}

The study design was a randomized, controlled trial conducted at four study sites in large metropolitan areas (Boston, Cleveland, New York City, and Washington, DC). Eligible participants completed an informed consent process, were randomized to one of two study arms, provided genetic risk information for $\mathrm{AD}$ by a study clinician (typically a genetic counselor, although physicians with expertise in $A P O E$ and $\mathrm{AD}$ also participated in some risk disclosure sessions), and were followed at multiple time points up to 1 year to monitor response to risk information. More details on study participants and procedures are provided in the following.

\section{Participants}

Adults with one living or deceased first-degree relative affected by late-onset $\mathrm{AD}$ (onset $\geq 60$ years) were eligible for participation. Individuals who had more than one affected first-degree relative, or whose affected family members' average age of $\mathrm{AD}$ onset was $<60$ years, were excluded. Participants were also excluded if they scored in clinically significant ranges on validated measures of cognitive functioning, depression, or anxiety. Participants were self-referred, having heard about the study through various sources including the Internet, community outreach events, word of mouth, or through other research studies at the study sites.

A total of 437 individuals expressed initial interest in the study. Of these, 131 declined participation in the trial due to a variety of reasons (e.g., yearlong study protocol was too burdensome, had concerns about the value of information provided). In addition, 14 did not meet family history eligibility criteria, and 12 were screened out because of scores on cognitive, mood, or anxiety scales that did not meet predetermined study criteria. The resulting 280 participants received AD risk assessment; 277 completed the 6-week follow-up visit; and 273 completed the 6-month follow-up visit. Of the 280 participants who received $\mathrm{AD}$ risk assessment, 16 were excluded from analysis because they did not complete the main study outcome measures of risk recall and comprehension. Final analyses were conducted on the remaining 264 participants.

\section{Procedures}

An outline of the study appears in Table 1. Demographic information was ascertained during a brief intake interview conducted by telephone, and a follow-up mail survey assessed variables including numeracy and baseline knowledge about AD. All participants provided informed consent at the time of initial study enrollment. Individuals who chose to enroll in the trial were then randomized to one of two $\mathrm{AD}$ genetics education protocols. In both protocols, participants were provided information regarding $\mathrm{AD}$ risk factors, the relationship between $A P O E$ genotype and $\mathrm{AD}$ risk, and the potential risks and benefits of receiving genetic testing. However, participants in one arm (hereafter referred to as the Condensed Protocol, or CP) were educated via a four-page brochure (see Supplementary Methods and Procedures online) mailed to their home in advance of an in-person appointment, whereas participants in the other arm (hereafter referred to as the Extended Protocol, or EP) attended a separate in-person education session with the study clinician in which a structured PowerPoint presentation was given (see Table 1 for an overview of how procedures differed by study arm). Participants in both arms received information about the following topics: (i) a formal definition of $\mathrm{AD}$; (ii) general risk factors for $\mathrm{AD}$ (e.g., age, family history) and the general population's level of risk; (iii) APOE genotype and its implications for risk of $\mathrm{AD}$; (iv) procedures involved in APOE testing; (v) a preview of what would be provided in their risk assessment (e.g., risk figures and their format, comparison groups); and (vi) known benefits, risks, and limitations of $A P O E$ genotype testing (e.g., lack of precision in risk estimates, potential privacy concerns regarding genetic information). In the next phase of the study, participants met with the study clinician for a posteducation session in which some procedures were employed for all participants, irrespective of study arm. First, participants were screened for clinically significant cognitive impairment, anxiety, and depression. The study

\section{Table 1 Comparison of extended versus condensed protocol procedures}

\begin{tabular}{|c|c|c|c|}
\hline Session & Topics addressed & Extended Protocol & Condensed Protocol \\
\hline \multirow[t]{5}{*}{ Education } & General AD risk factors & In person & Brochure \\
\hline & $A P O E$ and risk of $A D$ & In person & Brochure \\
\hline & APOE testing procedures & In person & Brochure \\
\hline & Explanation of risk assessment & In person & Brochure \\
\hline & Test benefits, risks, and limitations & In person & Brochure \\
\hline \multirow[t]{2}{*}{ Posteducation/blood draw } & Answering participant questions & Initiated by clinician & Initiated by clinician \\
\hline & Exploring psychosocial aspects & Initiated by clinician & Only if participant initiated \\
\hline Risk disclosure & $A P O E$ genotype result and $A D$ risk estimate & In person & In person \\
\hline \multicolumn{4}{|l|}{ Follow-up } \\
\hline 6 Weeks & Assessment of risk recall and risk knowledge & In person & In person \\
\hline 6 Months & Assessment of risk recall and risk knowledge & In person & In person \\
\hline
\end{tabular}

$A D$, Alzheimer disease; $A P O E$, gene encoding apolipoprotein $E$. 
clinician then reviewed the participant's personal medical history, including family history of $\mathrm{AD}$, and offered to review the educational materials and address any participant questions or concerns before continuing with the genetic testing. One distinction between the arms was that the EP included structured exploration of potential psychosocial issues involved for participants in testing, whereas these issues were addressed in the $\mathrm{CP}$ only upon the participants' initiative. In both arms, the last activity of this visit was a blood draw (participants were reconsented before collection of DNA), with blood samples sent to a Clinical Laboratory Improvement Amendments-approved laboratory for $A P O E$ genotype analysis.

Once genotype results were available, a subsequent in-person disclosure session was scheduled. Here, participants in both study arms received both their APOE genotype results and an estimated lifetime risk of developing $\mathrm{AD}$, defined as the cumulative risk of developing $\mathrm{AD}$ from birth to age of 85 years. The estimates were based on gender- and genotypespecific AD risk curves developed for African-American and white participants. ${ }^{7}$ Participants who carried at least one copy of the APOE $\& 4$ allele were given specific lifetime AD risk estimates ranging from 25 to $74 \%$. Individuals without an $\varepsilon 4$ allele received specific lifetime $\mathrm{AD}$ risk estimates ranging from 13 to $50 \%{ }^{8,9}$ In addition to their numeric risk estimates, participants also received genotype-specific graphic representation of their risk that compared their risk with (i) the general population and (ii) first-degree relatives of patients with $\mathrm{AD}$. A standard script was used to provide participants with a verbal explanation of their $\mathrm{AD}$ risk estimate and the personal information that was used to generate the risk estimate (i.e., their $A P O E$ genotype, family history, gender, race, and age). Participants also received a standardized, one-page written summary of the risk estimate and its limitations. Participants were asked to complete follow-up surveys at 6 weeks and 6 months following risk disclosure.

\section{Measures}

Demographics. Participants' age, gender, race, and level of education were assessed by standard self-report questionnaire items.

Numeracy. Objective numeracy skills (i.e., facility with numbers) were assessed using a validated eight-item scale developed by Lipkus et al. ${ }^{10}$ (sample item: "if the chance of getting a disease is $10 \%$, how many people would be expected to get the disease out of 100 people?"). Possible scores ranged from 0 to 8 (indicating number of items answered correctly), with higher scores indicating higher level of numeracy.

Length of time with study clinician. We estimated face-to-face time with the study clinician by recording the start and end times for the study's posteducation and risk disclosure sessions. For the EP, we estimated average length of time for the pretest education session-there was no such session in the CP-based on interviews with study clinicians and review of their chart notes (start and end times were not formally documented for this particular session).

Illness perceptions. At baseline, participants were asked about their self-perceived risk of $\mathrm{AD}$ and their beliefs about the controllability of AD. Perceived risk was assessed by the following item: "On a scale of $0-100 \%$, what do you believe your chances are of developing Alzheimer's disease sometime in your life?" (possible range: $0-100$ ). Perceived control over the development of $\mathrm{AD}$ was assessed via a four-item summed scale (possible range: 4-20, with higher scores indicating greater degree of perceived control) where questions measured the degree to which the participant believed the onset or severity of AD could be modified by personal actions.

Risk knowledge. Knowledge related to $A P O E$ genotype and other risk factors for $\mathrm{AD}$ was assessed both by items from validated AD knowledge scales ${ }^{11}$ and others created specifically for this study. Items created for this study were based on education material provided in the protocol and developed by a multidisciplinary team of investigators with expertise in $\mathrm{AD}$, epidemiology, genetics, and genetic counseling. In this analysis, our primary outcome measure was a four-item scale consisting of the following multiple-choice questions: (i) "What is the average person's lifetime risk of getting $\mathrm{AD}$ ?"; (ii) "How are people's chances of developing AD different if they have a parent or sibling who has had the disease?"; (iii) "Can the APOE genetic test predict with certainty whether or not a person will get AD?"; and (iv) "How does having an APOE $\varepsilon 4$ gene affect the chances that someone will get $\mathrm{AD}$ ?". The number of correct responses was summed to create an overall risk knowledge score (range: $0-4$ ), and this scale was administered at baseline, at the pretest blood draw session following genetic education, and 6 months following risk disclosure.

In addition, the following items were administered only at follow-up: (i) "Men are more likely to develop Alzheimer's disease than women" (true/false); (ii) "What form of APOE is the risk-increasing form?" (multiple choice); (iii) "Which of the following factors were used to calculate your personal risk assessment?" (multiple choice); (iv) "A genetic test result that does not include a copy of the risk-increasing form of the $A P O E$ gene means that there is $0 \%$ chance of developing $\mathrm{AD}$ " (true/false). These items allowed us to assess additional aspects of participants' knowledge of $\mathrm{AD}$ risk, as well as protocolspecific information that would not likely have been known at baseline.

Risk recall. Recall of risk information was assessed by four items, each administered 6 weeks and 6 months following disclosure. In open-ended response items, participants were asked to recall the lifetime risk estimates they were provided. Following procedures used in other studies of risk recall, ${ }^{12-14}$ we considered an answer correct for each of these items if it was within five points of the actual risk estimate disclosed. In addition, participants were asked to identify their $A P O E$ genotype from a list 
of six possible genotypes. Participants were also asked to note whether they carried 0,1 , or 2 copies of the "risk-increasing form (allele) of APOE."

\section{Data analyses}

Descriptive statistics were used to characterize the sample in terms of its demographic characteristics and scores on outcome measures. $\chi^{2}$ analyses and $t$-tests were used to assess differences between EP and CP participants on knowledge and recall, including both individual scale items and summed scale scores (where scores equaled number of items answered correctly in each domain). Repeated-measures analyses of variance were used to assess $\mathrm{EP}$ versus $\mathrm{CP}$ group differences while accounting for the effects of key demographic variables and other characteristics that have been suggested by prior research to be associated with knowledge and recall; these included age, gender, race (white versus other), education level, numeracy, time, baseline self-perceived risk, perceived $\mathrm{AD}$ control, and $A P O E$ status. Multivariate repeated-measures analyses adjusted for the aforementioned variables as well as time. All statistical calculations were performed using SAS version 9.2 (Cary, NC).

\section{RESULTS}

\section{Sample characteristics}

Overall, the sample had a mean age of 58 years, with the majority of participants being female (71\%) and Caucasian (80\%). The mean level of education among participants was 16 years, and the average score on the numeracy scale was 6.9 items out of 8 answered correctly. Forty-two percent of participants carried at least one $\varepsilon 4$ allele. The $\mathrm{EP}$ and $\mathrm{CP}$ study arms did not differ significantly in terms of the demographics assessed. A full description of sample characteristics, stratified by study arm, is presented in Table 2.

\section{Length of time with study clinician}

Overall, CP participants averaged 34 min of time with the study clinician across two in-person sessions, whereas EP participants averaged $77 \mathrm{~min}$ of time across three sessions. In the CP arm, post-education sessions averaged $13 \mathrm{~min}(\mathrm{SD}=8.9)$ and risk disclosure session averaged $21 \mathrm{~min}(\mathrm{SD}=14.4)$. In the EP arm, post-education sessions averaged $20 \mathrm{~min}(\mathrm{SD}=8.9)$ and risk disclosure sessions averaged $22 \mathrm{~min}(\mathrm{SD}=10.6)$, with the education sessions unique to the EP arm estimated by study clinicians to have taken $35 \mathrm{~min}$ on average.

\section{Risk knowledge}

Participants in both study arms improved from baseline in terms of their risk knowledge scores. The average score among $\mathrm{CP}$ participants was 3.2 at post-education as compared with 2.4 at baseline $(P<0.0001)$, and the average score for EP participants was 3.4 at posteducation as compared with 2.3 at baseline $(P<0.0001)$. There was no statistical difference in either group between mean risk knowledge scores at posteducation versus 6-month follow-up (CP: 3.2 at posteducation and 3.2 at 6 months, $P=0.95$; EP: 3.4 at post-education and 3.4 at 6 months,
Table 2 Sample characteristics

\begin{tabular}{|c|c|c|c|}
\hline Characteristic & $\begin{array}{l}\text { Extended } \\
\text { Protocol } \\
(N=83)\end{array}$ & $\begin{array}{c}\text { Condensed } \\
\text { Protocol } \\
(N=181)\end{array}$ & $P$ value \\
\hline \multicolumn{4}{|l|}{ Age, years } \\
\hline Mean (SD) & $57.6(10.5)$ & $58.3(10.7)$ & 0.61 \\
\hline Range & $36-78$ & $33-86$ & \\
\hline \multicolumn{4}{|l|}{ Gender } \\
\hline Female & $58(69.9 \%)$ & $130(71.8 \%)$ & 0.75 \\
\hline Male & $25(30.1 \%)$ & $51(28.2 \%)$ & \\
\hline \multicolumn{4}{|l|}{ Years of education } \\
\hline Mean (SD) & $16.3(2.6)$ & $16.1(2.5)$ & 0.49 \\
\hline Range & $10-20$ & $3-20$ & \\
\hline \multicolumn{4}{|l|}{ Race } \\
\hline Caucasian & $65(78.3 \%)$ & $146(80.7 \%)$ & 0.66 \\
\hline Non-Caucasian & $18(21.7 \%)$ & $35(19.3 \%)$ & \\
\hline \multicolumn{4}{|l|}{ Objective numeracy } \\
\hline Mean score (SD) & $6.9(1.7)$ & $7.0(1.5)$ & 0.82 \\
\hline Range & $1-8$ & $0-8$ & \\
\hline \multicolumn{4}{|c|}{ Baseline self-perceived risk } \\
\hline Mean rating (SD) & $51.4(24.0)$ & $50.6(23.0)$ & 0.80 \\
\hline Range & $0-100$ & $0-100$ & \\
\hline \multicolumn{4}{|l|}{ Perceived AD control } \\
\hline Mean score (SD) & $12.5(2.9)$ & $12.5(3.0)$ & 0.93 \\
\hline Range & $4-19$ & $4-19$ & \\
\hline \multicolumn{4}{|l|}{ APOE status } \\
\hline No $\varepsilon 4$ alleles & $53(63.9 \%)$ & $101(55.8 \%)$ & 0.22 \\
\hline One $\varepsilon 4$ allele & $27(32.5 \%)$ & $70(38.7 \%)$ & \\
\hline Two $\varepsilon 4$ alleles & $3(3.6 \%)$ & $10(5.5 \%)$ & \\
\hline
\end{tabular}

The vast majority of the non-Caucasian group were African American (18.6\% of total sample).

$A D$, Alzheimer disease; $A P O E$, gene encoding apolipoprotein $E$.

$P=0.93)$. Analyses of individual survey items showed no statistical differences between study arms on any items at the posteducation time point; at 6-month follow-up, differences were found at the $P<0.05$ level for two items (see Table 3 ) but these items were no longer significant when applying a prespecified $P<0.01$ criterion to account for multiple comparisons. Table 3 presents a full description of participants' performance on individual risk knowledge items.

\section{Risk recall}

Overall, participants in both study arms scored a mean of 2.4 items correct out of 4 on the risk recall measure. Overall mean scores did not differ by study arm at either time point, and there were no differences in either group on risk recall at the 6-month versus 6-week follow-up (i.e., no significant effects of time on recall). Table 4 presents a full description of participants' performance on individual risk recall items, and Table 5 shows results from a multivariate repeated-measures model 
Table 3 Respondents' percentage of correct responses on risk knowledge items, by study arm and time point

\begin{tabular}{|c|c|c|c|c|}
\hline Item (response choices) & Study arm & Baseline (\%) ${ }^{a}$ & $\begin{array}{l}\text { Posteducation } \\
\quad(\%)\end{array}$ & $\begin{array}{l}\text { Six-month follow-up } \\
(\%)^{\mathrm{a}}\end{array}$ \\
\hline $\begin{array}{l}\text { What is the average person's lifetime risk of getting AD? } \\
(>75 \%, 45-50 \%, 10-15 \%, 1-5 \%)\end{array}$ & Extended & 48 & 75 & 83 \\
\hline $\begin{array}{l}\text { How are chances of developing AD different if a parent or sibling } \\
\text { has had AD? (no difference, somewhat higher, a lot higher) }\end{array}$ & Extended & 68 & 86 & 83 \\
\hline \multirow{2}{*}{$\begin{array}{l}\text { Can } A P O E \text { predict with certainty whether someone will get } A D \text { ? } \\
(y e s, \mathbf{n o})\end{array}$} & Extended & 55 & 87 & 81 \\
\hline & Condensed & 53 & 82 & 82 \\
\hline \multirow{2}{*}{$\begin{array}{l}\text { How does having APOE- } 4 \text { affect one's chances of } A D \text { ? (makes it } \\
\text { practically certain to not get } A D \text {, makes it somewhat less likely, } \\
\text { makes it somewhat more likely, makes it practically certain) }\end{array}$} & Extended & 60 & 90 & 89 \\
\hline & Condensed & 57 & 83 & 91 \\
\hline \multirow[t]{2}{*}{ What form of $A P O E$ is risk increasing? $(\varepsilon 2, \varepsilon 3, \varepsilon 4)$} & Extended & - & 73 & 67 \\
\hline & Condensed & - & 67 & 53 \\
\hline \multirow{2}{*}{$\begin{array}{l}\text { What factors are used in your personal risk assessment (age, gender, } \\
A P O E \text { results, all of the above) }\end{array}$} & Extended & - & 93 & 86 \\
\hline & Condensed & - & 84 & 83 \\
\hline \multirow{2}{*}{$\begin{array}{l}\text { A genetic test result that is not } A P O E \& 4 \text { means } 0 \% \text { chance of } A D \\
\text { (true, false) }\end{array}$} & Extended & - & 98 & 96 \\
\hline & Condensed & - & 95 & 96 \\
\hline
\end{tabular}

Boldface values represent correct answers. $A D$, Alzheimer disease; $A P O E$, gene encoding apolipoprotein $E$.

aThere were significant group differences on bolded items at the $P<0.05$ level, but differences were not significant when using a $P<0.01$ level to adjust for multiple comparisons.

predicting both risk knowledge and risk recall composite score outcomes.

\section{DISCUSSION}

Genomic medicine may increasingly involve the use of genotyping to help estimate risk of common complex diseases. The traditional, time-intensive model of genetic education and counseling for rare heritable diseases will not be sustainable for such risk assessment given the high prevalence of common diseases and the limited number of genetic specialists available. Our results in the context of AD suggest the viability of a more abbreviated model for providing education and genetic susceptibility testing to at-risk individuals. The substitution of written education materials for an initial in-person education session, coupled with a more streamlined approach to provision of information, resulted in a protocol requiring fewer sessions and significantly less face-to-face time with study clinicians.

This reduction in time with the clinician was not correlated with lower levels of knowledge or poorer recall of key $\mathrm{AD}$ risk information. Both groups achieved significant gains from baseline in terms of knowledge of basic facts about $\mathrm{AD}$ risk. In addition, the majority of participants in both groups recalled pertinent personal risk information and retained it
Table 4 Respondents' percentage of correct responses on risk recall items at 6-week and 6-month follow-up, by study arm

\begin{tabular}{llcc} 
Item & Study arm & $\begin{array}{c}\text { Six-week } \\
\text { follow-up }\end{array}$ & $\begin{array}{c}\text { Six-month } \\
\text { follow-up }\end{array}$ \\
\hline $\begin{array}{llcc}\text { Lifetime risk of AD } \\
\text { (within 5 points; \%) }\end{array}$ & Extended & 61 & 62 \\
Remaining risk of AD & Condensed & 66 & 68 \\
(within 5 points; \%) & Extended & 71 & 64 \\
\hline Exact genotype recall (\%) & Condensed & 74 & 62 \\
& Extended & 66 & 62 \\
\hline Recall of APOE \&4 status (\%) & Condensed & 59 & 58 \\
& Extended & 58 & 65 \\
& Condensed & 60 & 66 \\
\hline
\end{tabular}

There were no significant group differences on any of these items. AD, Alzheimer disease; $A P O E$, gene encoding apolipoprotein $\mathrm{E}$.

over time, at rates generally higher than seen at comparable time points in studies of risk perception accuracy following genetic counseling ${ }^{15,16}$ (although it should be noted that significant proportions of participants in both groups did not retain certain details from their risk disclosure sessions; for example, nearly half of $\mathrm{CP}$ participants could not recall at 6 
Table 5 Multivariate repeated-measures analysis of group differences in risk knowledge and recall

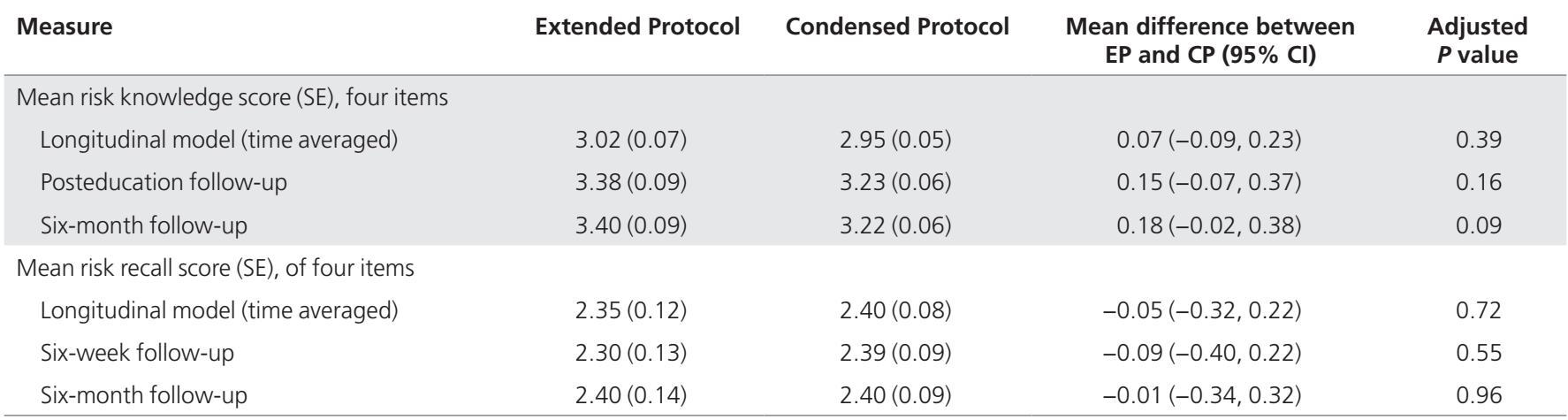

Scores are adjusted for age, gender, race, education level, objective numeracy, baseline self-perceived risk, perceived AD control, and $A P O E \varepsilon 4$ status.AD, Alzheimer disease; $A P O E$, gene encoding apolipoprotein $\mathrm{E} ; \mathrm{Cl}$, confidence interval; $C P$, Condensed Protocol; EP, Extended Protocol.

months which $A P O E$ allele was associated with increased $\mathrm{AD}$ risk). The condensed approach may have benefited from a "less is more" approach by focusing on information deemed to be most salient to participants and eliminating extraneous material. Genetic education approaches may be enhanced by reducing the time spent on review of the basic principles of genetics, in favor of more personalized, "take-home" messages regarding the individual's clinical situation. ${ }^{17}$

This study adds to the small but growing literature on the educational efficacy of brief, alternative approaches to genetic education and counseling for adult-onset disorders. Much of this work has been done in the context of BRCA $1 / 2$ testing for risk of hereditary breast/ovarian cancer. One example is the landmark study by Green et al. ${ }^{18,19}$ of an interactive computer program developed for use in genetic counseling programs for breast cancer, with satisfactory patient education and satisfaction outcomes. A related randomized clinical trial involving a supplementary CD-ROM education tool suggested that this intervention resulted in less face-to-face time with the genetics team and lower likelihood of cancerrelated worry following testing. ${ }^{20}$ More recently, Graves and colleagues ${ }^{21}$ found that telephone counseling can serve as an effective adjunct to standard in-person counseling for BRCA testing, and Jenkins and colleagues ${ }^{23}$ found that phone and in-person approaches achieved similar results in terms of patient knowledge. ${ }^{22}$

Of course, it should be noted that there are certain situations where in-person sessions and/or more extended time with study clinicians would be appropriate or even essential (e.g., disclosure of genetic testing results for Huntington disease). Our focus here was on risk information knowledge and recall, but responses of interest in genetic education and counseling include psychosocial adjustment and health behaviors, outcomes that were beyond the scope of this particular study (the larger REVEAL study will address some of these outcomes in forthcoming articles). It should also be noted that our findings cannot be generalized to emerging genetic susceptibility testing options provided by personal genomics service companies, where education and counseling are provided via website alone and/or with telephone counseling. ${ }^{24-26}$
This study had several limitations that should be kept in mind when interpreting study results. Most important, the study sample was relatively small and biased toward participants with high levels of education and socioeconomic status. In addition, participants were familiar with the condition in question, highly numerate, clinically stable, and generally highly motivated to receive genetic risk information. It is unclear whether our results would be generalizable to populations that differ on the aforementioned characteristics. Our measures of session length for the education session in the EP relied on retrospective estimates (versus real-time assessment) from the study clinicians, which likely resulted in imprecise assessment. In addition, not all items that assessed risk recall and knowledge were formally validated, and the repeated administration of these measures may have resulted in practice effects and/or between-session review of results that artificially inflated the rates of correct responses at the 6-month follow-up. Finally, although study measures of knowledge and recall had high face validity, their psychometric properties are not well established.

There are numerous important avenues for future research on this topic. An important first step would be to see if briefer protocols can be successfully employed with diverse populations, especially groups with lower levels of education and health literacy than seen in our study. Such studies would do well to draw upon advances in the medical decision-making literature, where techniques are being developed to simplify descriptions of health issues and options, provide effective decision support tools, and enhance communication of genetic and other types of risk information. ${ }^{27-29}$ The rapid pace of progress in wholegenome sequencing may mean that genomic education will need to address multiplex (as opposed to single-gene) testing, which will only complicate the challenges at hand for the health educator. ${ }^{30}$ Studies that examine the provision of numerous genetic test results at once, as well as the potential pleiotropic effects of genetic markers, may be required to guide practice in this emerging area. Current trends in genomic medicine also suggest that services will need to be delivered by generalists (e.g., primary-care practitioners), ${ }^{1}$ and thus research involving a broader range of health professionals would be advisable. As alluded to earlier, in evaluating the efficacy of education 
and counseling programs, it will be important to look beyond merely the recall and comprehension of information provided to assess how people use their test results and how they cope with the implications of risk disclosure for themselves and their family members.

\section{SUPPLEMENTARY MATERIAL}

Supplementary material is linked to the online version of the paper at http://www.nature.com/gim

\section{ACKNOWLEDGMENTS}

This work was supported by grants from the National Human Genome Research Institute (HG02213, HG005092). We acknowledge members of the REVEAL Study Group, including teams at Boston University (Adrienne Cupples, Lindsay Farrer), Case Western Reserve University (Peter Whitehouse and Melissa Barber Butson), Weill Medical College of Cornell University (Norman Relkin), Duke University (Robert Cook-Deegan, Charmaine Royal), Howard University (Thomas Obisesan, Grace Fasaye), and the University of Michigan (Kurt Christensen, Erin Linnenbringer). We also thank Lan Le for her assistance with manuscript preparation and Kurt Christensen for his helpful feedback on an earlier version of the manuscript.

\section{DISCLOSURE}

The authors declare no conflict of interest.

\section{REFERENCES}

1. American Board of Genetic Counselors website. www.abgc.net/ABGC/ AmericanBoardofGeneticCounselors.asp. Accessed 23 March 2012.

2. Guttmacher AE, Jenkins J, UhImann WR. Genomic medicine: who will practice it? A call to open arms. Am J Med Genet 2001;106:216-222.

3. Guttmacher AE. Human genetics on the web. Annu Rev Genomics Hum Genet 2001;2:213-233.

4. Cupples LA, Farrer LA, Sadovnick AD, Relkin N, Whitehouse P, Green RC. Estimating risk curves for first-degree relatives of patients with Alzheimer's disease: the REVEAL study. Genet Med 2004;6:192-196.

5. Roberts JS, Barber M, Brown TM, et al. Who seeks genetic susceptibility testing for Alzheimer's disease? Findings from a multisite, randomized clinical trial. Genet Med 2004;6:197-203.

6. LaRusse S, Roberts JS, Marteau TM, et al. Genetic susceptibility testing versus family history-based risk assessment: Impact on perceived risk of Alzheimer disease. Genet Med 2005;7:48-53.

7. Eckert SL, Katzen H, Roberts JS, et al. Recall of disclosed apolipoprotein E genotype and lifetime risk estimate for Alzheimer's disease: the REVEAL Study. Genet Med 2006;8:746-751.

8. Christensen KD, Roberts JS, Royal CD, et al. Incorporating ethnicity into genetic risk assessment for Alzheimer disease: the REVEAL study experience. Genet Med 2008;10:207-214

9. Chao S, Roberts JS, Marteau TM, Silliman R, Cupples LA, Green RC. Health behavior changes after genetic risk assessment for Alzheimer disease: The REVEAL Study. Alzheimer Dis Assoc Disord 2008;22:94-97.
10. Lipkus IM, Samsa G, Rimer BK. General performance on a numeracy scale among highly educated samples. Med Decis Making 2001;21:37-44.

11. Moscarillo TJ, Holt H, Perman M, et al. Knowledge of and attitudes about Alzheimer disease genetics: report of a pilot survey and two focus groups. Community Genet 2007;10:97-102.

12. Cull A, Miller $H$, Porterfield $T$, et al. The use of videotaped information in cancer genetic counselling: a randomized evaluation study. Br J Cancer 1998;77:830-837.

13. Evans DG, Blair V, Greenhalgh R, Hopwood P, Howell A. The impact of genetic counselling on risk perception in women with a family history of breast cancer. Br J Cancer 1994;70:934-938.

14. Lerman C, Lustbader E, Rimer B, et al. Effects of individualized breast cancer risk counseling: a randomized trial. J Natl Cancer Inst 1995;87:286-292.

15. Smerecnik CM, Mesters I, Verweij E, de Vries NK, de Vries H. A systematic review of the impact of genetic counseling on risk perception accuracy. J Genet Couns 2009;18:217-228.

16. Meiser B, Halliday JL. What is the impact of genetic counselling in women at increased risk of developing hereditary breast cancer? A meta-analytic review. Soc Sci Med 2002;54:1463-1470.

17. Julian-Reynier C, Welkenhuysen M, Hagoel L, Decruyenaere M, Hopwood P; CRISCOM Working Group. Risk communication strategies: state of the art and effectiveness in the context of cancer genetic services. Eur J Hum Genet 2003;11:725-736.

18. Green MJ, Biesecker BB, McInerney AM, Mauger D, Fost N. An interactive computer program can effectively educate patients about genetic testing for breast cancer susceptibility. Am J Med Genet 2001;103:16-23.

19. Green MJ, Peterson SK, Baker MW, et al. Effect of a computer-based decision aid on knowledge, perceptions, and intentions about genetic testing for breast cancer susceptibility: a randomized controlled trial. JAMA 2004;292:442-452.

20. Wang C, Gonzalez R, Milliron KJ, Strecher VJ, Merajver SD. Genetic counseling for BRCA1/2: a randomized controlled trial of two strategies to facilitate the education and counseling process. Am J Med Genet $A$ 2005;134A:66-73.

21. Graves KD, Wenzel L, Schwartz MD, et al. Randomized controlled trial of a psychosocial telephone counseling intervention in BRCA1 and BRCA2 mutation carriers. Cancer Epidemiol Biomarkers Prev 2010;19:648-654.

22. Peshkin BN, Demarco TA, Graves KD, et al. Telephone genetic counseling for high-risk women undergoing BRCA1 and BRCA2 testing: rationale and development of a randomized controlled trial. Genet Test 2008;12:37-52.

23. Jenkins J, Calzone KA, Dimond E, et al. Randomized comparison of phone versus in-person BRCA1/2 predisposition genetic test result disclosure counseling. Genet Med 2007;9:487-495.

24. Hunter DJ, Khoury MJ, Drazen JM. Letting the genome out of the bottle-will we get our wish? N Engl J Med 2008;358:105-107.

25. Wolfberg AJ. Genes on the Web-direct-to-consumer marketing of genetic testing. N Eng/ J Med 2006;355:543-545.

26. Offit K. Genomic profiles for disease risk: predictive or premature? JAMA 2008;299:1353-1355.

27. Sheridan SL, Halpern DJ, Viera AJ, Berkman ND, Donahue KE, Crotty K. Interventions for individuals with low health literacy: a systematic review. J Health Commun 2011;16(suppl 3):30-54.

28. Stacey D, Bennett CL, Barry MJ, et al. Decision aids for people facing health treatment or screening decisions. Cochrane Database Syst Rev 2011; CD001431.

29. Food and Drug Administration. Communicating Risks and Benefits: An Evidence-Based User's Guide. US Department of Health and Human Services: Silver Springs, MD, 2011.

30. McBride CM, Alford SH, Reid RJ, Larson EB, Baxevanis AD, Brody LC. Putting science over supposition in the arena of personalized genomics. Nat Genet 2008;40:939-942. 